\title{
Factores asociados al consumo de Tabaco en Adolescentes
}

\author{
Alejandro Villena Ferrer ${ }^{a}$, Susana Morena Rayo ${ }^{b}$, Ignacio Párraga Martínez ${ }^{c}$,

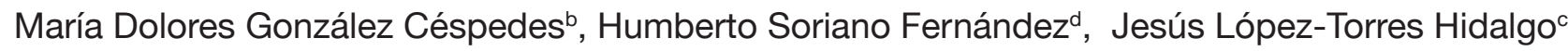

\begin{abstract}
a Médico de Familia. Centro de Salud Zona V-B de Albacete.

${ }^{\mathrm{b}}$ Médico de Familia. Centro de Salud Hellín II, Hellín (Albacete).
\end{abstract}

\section{c Médico de Familia.} Unidad de Investigación de la Gerencia de Atención Primaria de Albacete.

\author{
d Médico de Familia. \\ Centro de Salud de Casas \\ de Juan Núñez (Albacete).
}

Correspondencia: Alejandro Villena Ferrer, Centro de Salud Zona V-B, c/ Macedonio Jiménez $\mathrm{s} / \mathrm{h}$, 02006-Albacete.

Telf.: 967510174, e-mail: avillenaferrer@papps.org

Recibido el 19 de enero de 2009.

Aceptado para su publicación el 23 de febrero de 2009.

\section{RESUMEN}

Objetivo. Identificar factores asociados al consumo de tabaco en escolares de $1^{\circ}$ de ESO.

Diseño. Estudio analítico de casos y controles apareados por sexo (razón 1:3).

Emplazamiento. Centros escolares pertenecientes a 16 zonas básicas de salud de Albacete y Ciudad Real.

Participantes. Escolares de $1^{\circ}$ de ESO seleccionados mediante cuestionario autocumplimentado. En la muestra participaron 146 escolares fumadores (casos) y 438 no fumadores (controles).

Mediciones principales. Variables sobre consumo de tabaco en el entorno sociofamiliar, rendimiento escolar, consumo de alcohol, práctica deportiva y otras relacionadas con estilos de vida.

Resultados. En los adolescentes fumadores fue significativamente superior $(p<0,001)$ la proporción de padres fumadores y con menor nivel de estudios, así como la de hermanos, amigos o profesores fumadores. El consumo de alcohol, la ausencia de práctica deportiva, mayor edad, mayor disponibilidad de dinero o menor número de asignaturas aprobadas fueron otros factores asociados $(p<0,01)$. Mediante regresión logística, las variables asociadas independientemente fueron: amigos fumadores (OR:11,3; IC95\%:4,2-30,9), consumo de alcohol (OR:6,9; IC95\%:3,1-15,1), ausencia de práctica deportiva (OR:3,3; IC95\%:1,4-7,6), mayor edad (14-15 años) (OR:2,3; IC95\%:1,2-4,6) y menor nivel de estudios del padre (OR:2,0; IC95\%:1,1-3,9).

Conclusiones. En el consumo de tabaco en adolescentes se identifican factores de exposición relacionados con el estilo de vida, tanto personal como de padres o amigos, entre ellos la presencia de fumadores en el entorno sociofamiliar o la menor dedicación a prácticas deportivas. Por otra parte, pueden ser también factores de riesgo algunas variables sociodemográficas como el menor nivel de estudios de los padres o la mayor edad del joven.

Palabras clave. Tabaquismo. Adolescente. Factores de riesgo. Salud escolar. Trastornos relacionados con sustancias.

\section{ABSTRACT}

\section{Factors associated with smoking in adolescents}

Objective. To identify factors associated with smoking in secondary school children.

Design. Case-control analytical study paired by gender (ratio1:3)

Setting. Secondary schools from 16 health areas of Albacete and Ciudad Real.

Subjects. Secondary school children divided in two groups based on a self-completed questionnaire. Case group with 146 smokers and control group with 438 non smokers.

Main measurements. Variables related to smoking in the socio-family setting, academic results, alcohol consumption, participation in sports and lifestyle.

Results. The percentage of smoking parents and of lower parental educational status were significantly higher $(p<0.001)$ in the smoking group. The percentages of smoking siblings, smoking friends and smoking teachers were also higher in this group. Alcohol consumption, non-participation in sports, older age, greater availability of money or fewer passed subjects were other factors associated $(p<0.01)$ with the smoking group. By logistic regression, the independent associated variables were: smoking friends (OR:11.3; 95\% Cl: 4.2-30.9), alcohol consumption (OR:6.9; 95\% Cl:3.1-15.1), nonparticipation in sports (OR:3.3; 95\% Cl:1.4-7.6), older age (14-15 years old) (OR:2.3; 95\% Cl:1.2-4.6) and lower paternal educational status (OR:2.0; $95 \% \mathrm{Cl}: 1.1-3.9)$

Conclusions. Smoking in secondary school children is correlated with risk factors related to personal, parental and friends' lifestyles, such as smokers in socio-family setting or non- participation in sports. In addition, some socio-demographic variables could be also be risk factors such as lower parental educational status or older age of the children.

Key words. Smoking. Adolescent. Risk Factors. School Health. Substance-Related Disorders.

Esta investigación fue presentada en el VI Congreso Nacional de Tabaquismo, celebrado en Logroño durante los días 26 a 28 de Octubre de 2006. 


\section{INTRODUCCIÓN}

El consumo de tabaco constituye, en los países desarrollados, la primera causa de mortalidad y morbilidad en adultos que se podría prevenir. Se calcula que el tabaco provoca la muerte de unos 5 millones de personas al año en el mundo y de más de 50.000 personas en España. En los países desarrollados, el tabaco es responsable del $24 \%$ de todas las muertes entre hombres y del $7 \%$ entre las mujeres, aunque esta última cifra está aumentando como consecuencia de la incorporación de la mujer a esta adicción. La pérdida de esperanza de vida entre los fumadores es de 14 años de media, dato al que habría que añadir la calidad de vida perdida para resaltar fielmente la importancia de esta enfermedad adictiva ${ }^{1}$.

En España el gasto sanitario originado por sólo 6 enfermedades relacionadas con el tabaco asciende a 7.000 millones de euros anuales y, según datos del Banco Mundial, en los países desarrollados el gasto sanitario relacionado con el tabaco supone un porcentaje del 6 al $15 \%$ del gasto sanitario global ${ }^{1}$.

Pero el tabaquismo también origina mortalidad y morbilidad en los fumadores pasivos, es decir, en aquellas personas expuestas de forma involuntaria al humo del tabaco en los hogares o en los lugares públicos fundamentalmente. El humo del tabaco está catalogado por la Organización Mundial de la Salud y la Agencia Estadounidense de Protección del Medioambiente como carcinógeno de grado $\mathrm{A}$, es decir, que carece de un nivel seguro de exposición. La exposición al aire contaminado por humo del tabaco (ACHT) supone un riesgo de muerte por cáncer de pulmón de 1/1000 y un riesgo de muerte por enfermedad coronaria de 1/100. Las estimaciones más optimistas señalan que en Europa pueden fallecer unas 50.000 personas que no fuman cada año por exposición involuntaria al humo del tabaco? ${ }^{2}$.

Todas estas consecuencias derivadas de la conducta de fumar podrían prevenirse si se consiguiera evitar esta conducta, la cual suele empezar en la adolescencia. La edad de iniciación y experimentación, donde el adolescente tiene el primer contacto con el tabaco, se sitúa alrededor de los 11 años y el consumo regular suele iniciarse a los 13 años en nuestro país ${ }^{3}$. Según datos de la Encuesta Estatal sobre Uso de Drogas entre estudiantes de Enseñanzas Secundarias publicada en 2007, la prevalencia de consumo experimental entre jóvenes de 14 a 18 años se situaba en 2006 en torno al $46,1 \%$ y la prevalencia de consumo diario en este mismo grupo etario era del $14,8 \%$. En la adolescencia se han descrito diversos factores personales, sociales y ambientales que contribuyen al inicio y al mantenimiento del tabaquismo ${ }^{3}$.

El objetivo del estudio ha sido identificar los factores asociados al consumo de tabaco población escolar de $1^{\circ}$ de Enseñanza Secundaria Obligatoria (E.S.O.).

\section{MATERIAL Y MÉTODO}

Se trata de un estudio analítico de casos y controles apareados por sexo (razón 1:3), realizado en 21 colegios e institutos de enseñanza secundaria pertenecientes a 16 zonas básicas de salud de las provincias de Albacete y Ciudad Real. La encuesta se realizó en el marco de una actividad de prevención del tabaquismo en centros escolares que expresaron su consentimiento para participar a través de su Departamento de Orientación, Jefatura de Estudios o Dirección durante el curso 2005-2006.

Los participantes fueron jóvenes escolarizados en $1^{\circ}$ de ESO, etapa escolar que incluye habitualmente la población adolescente de 11 y 12 años, aunque pueden incluirse jóvenes de edades superiores que no han conseguido superar las pruebas necesarias para pasar al curso siguiente. Cada uno de los participantes cumplimentó, al inicio del estudio, un cuestionario que recogía de forma anónima su condición de fumador o de no fumador. Se consideraron fumadores a todos aquellos que manifestaban fumar de manera regular aunque la frecuencia fuera baja y no lo practicaran a diario. Aquellos escolares que declararon haber probado el tabaco en alguna ocasión, pero no haber continuado el consumo por no haberles gustado la experiencia se incluyeron entre los no fumadores. En base a esta característica se seleccionaron 2 grupos: uno constituido por 146 escolares fumadores que participaron como casos (todos los fumadores) y otro formado por 438 no fumadores que participaron como controles (seleccionados a partir de los alumnos no fumadores). Los casos y controles fueron apareados por sexo en razón 1:3.

Además del consumo de tabaco, el cuestionario entregado para su autocumplimentación incluía variables como: edad, sexo, edad de primer contacto con el tabaco, asignaturas aprobadas en el último periodo de evaluación, nivel de estudios de los padres, hábito tabáquico de los padres, hermanos, amigos, compañeros, profesores y otros trabajadores de su centro escolar, disponibilidad de dinero y gasto en tabaco en caso de fumadores, consumo de alcohol, práctica deportiva habitual, y recordar haber visto u oído publicidad del tabaco en el último año. Finalmente la encuesta contenía preguntas para determinar los conocimientos que poseían los participantes sobre los efectos del tabaco en la salud y también para conocer su opinión y actitud personal frente al consumo.

Los datos fueron analizados con el programa SPSS 15.0, utilizando la prueba $\chi^{2}$ para comprobar la asociación entre el consumo de tabaco y cada una de las variables consideradas. Una vez detectadas las 


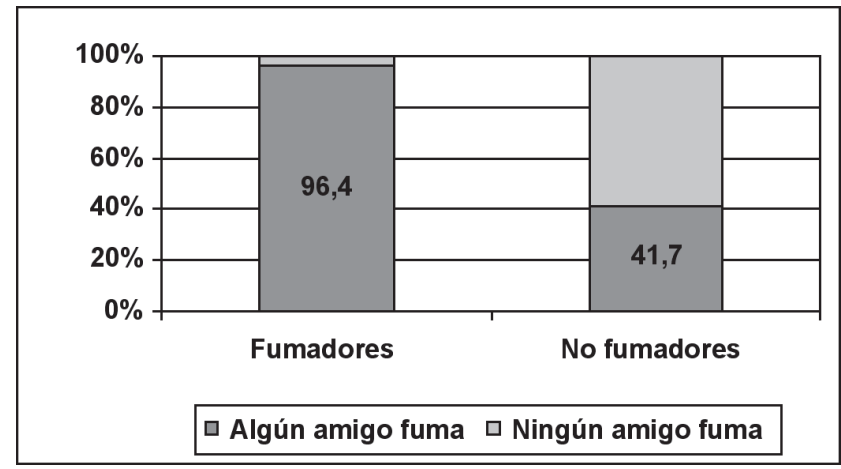

Figura 1. Proporción de adolescentes fumadores y no fumadores que poseen algún amigo fumador.

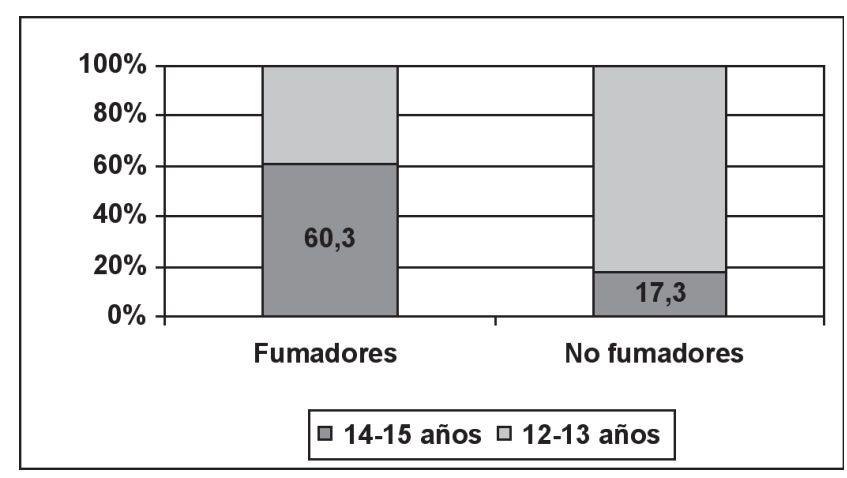

Figura 3. Proporción de fumadores y no fumadores según la edad

variables relacionadas con el consumo de tabaco, se realizó un análisis multivariante, mediante regresión logística, para determinar las variables que se asociaban de manera independiente al hábito de fumar. Las variables se incluyeron en el modelo por el método "forward stepwise", construyéndose en cinco pasos la ecuación de regresión logística e incluyéndose las variables con mayor significación estadística según la prueba de Wald (criterio de salida: $p=0,10$ ).

\section{RESULTADOS}

La edad media de los participantes fue de 13,1 $\pm 0,7$ años, siendo varones el $52,2 \%$ y mujeres el $47,8 \%$. En cuanto al consumo de tabaco entre las personas que constituyen el entorno próximo de los participantes (familiares, amigos y profesores), observamos que el $61,5 \%$ de los chicos fumadores aseguraba que su padre fumaba habitualmente. Esta proporción era significativamente inferior $(p=0,001)$ entre los chicos que no fumaban (44,7\%). En el mismo sentido, la proporción de madres fumadoras era significativamente superior $(p=0,016)$ entre los adolescentes fumadores $(52,7 \%)$ que entre los no fumadores $(40,6 \%)$. En el caso de los hermanos, el $55,4 \%$ de los fumadores te-

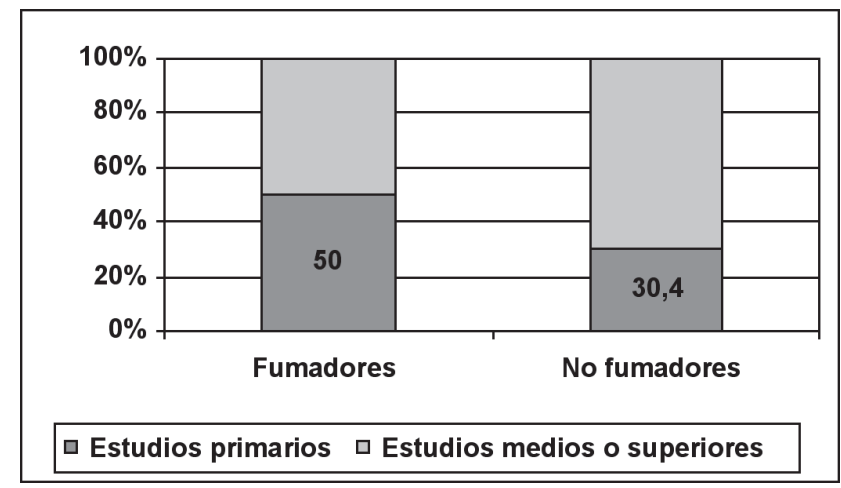

Figura 2. Nivel de estudios del padre en adolescentes fumadores y no fumadores.

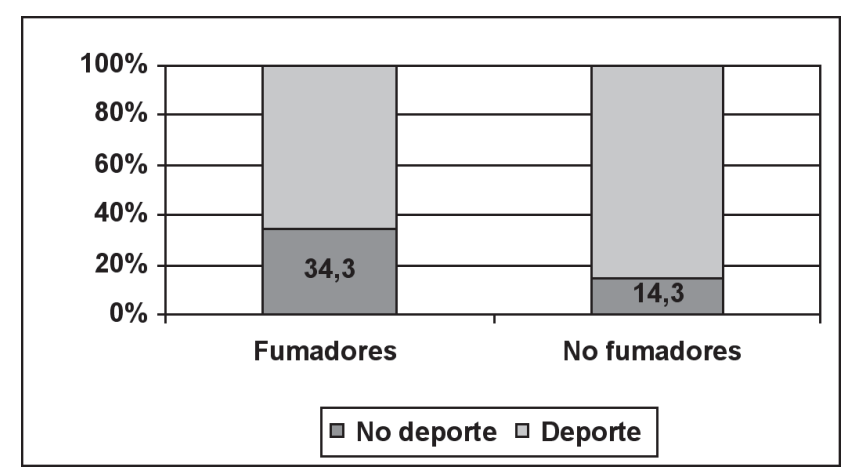

Figura 4. Proporción de fumadores y no fumadores según la práctica de deporte.

nía algún hermano fumador, proporción que descendía al 20,7\% entre los no fumadores ( $p<0,001)$. El 64,4 $\%$ de los chicos fumadores refería haber visto fumar a algún profesor, frente al $50,3 \%$ de los no fumadores $(p=0,013)$. Finalmente, la mayor diferencia encontrada se produjo en el caso de los amigos. En la figura 1 se puede observar que la proporción de adolescentes fumadores que contestaron afirmativamente cuando se les preguntaba si alguno de sus amigos fumaba era considerablemente mayor que la de no fumadores $(p<0,001)$.

Al analizar el nivel de estudios de los padres, se observó que, en el caso de la madre, el $47,1 \%$ de los encuestados fumadores declaraba que su madre no había pasado del nivel de estudios primario, mientras que esta proporción descendía al 31,2 \% entre los no fumadores $(p=0,003)$. En el caso del padre, las diferencias eran algo mayores, observándose que entre los chicos no fumadores la proporción de padres con estudios medios o superiores era superior $(p<0,001)$, tal y como se muestra en la figura 2.

Cuando se les preguntó por la cantidad de dinero que los padres o familiares les asignaban por semana, 


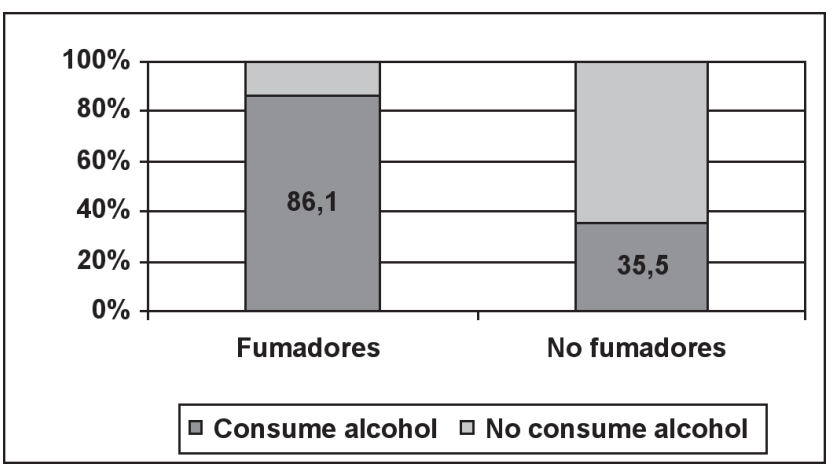

Figura 5. Proporción de fumadores y no fumadores en relación al consumo de alcohol

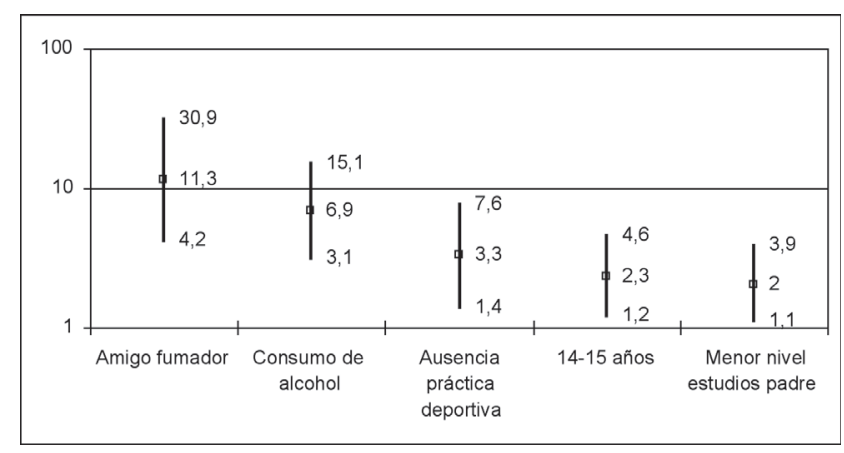

Figura 6. Representación comparativa de las variables asociadas independientemente al consumo de tabaco.

\begin{tabular}{|c|c|c|c|c|c|}
\hline Variable & Coeficiente & Wald & $\mathbf{p}$ & OR & IC95\% \\
\hline Amigo fumador & 2,433 & 22,787 & $<0,001$ & 11,3 & $4,2-30,9$ \\
\hline Consumo de alcohol & 1,932 & 23,358 & $<0,001$ & 6,9 & $3,1-15,1$ \\
\hline Ausencia de práctica deportiva & 1,204 & 8,133 & $<0,01$ & 3,3 & $1,4-7,6$ \\
\hline Edad de 14 y 15 años & 0,852 & 6,274 & $<0,05$ & 2,3 & $1,2-4,6$ \\
\hline Menor nivel de estudios del padre & 0,694 & 4,009 & $<0,05$ & 2,0 & $1,1-3,9$ \\
\hline
\end{tabular}

Tabla 1. Variables asociadas de manera independiente al consumo de tabaco en el análisis multivariante.

observamos que el $57,8 \%$ de los fumadores disponía de más de 6 euros por semana, mientras que esa cantidad sólo era alcanzada por el $21,3 \%$ de los no fumadores $(p<0,001)$.

La edad fue otro de los factores asociados al consumo de tabaco. En este sentido se observó que la proporción de chicos con 14 y 15 años era superior entre los fumadores $(p<0,001)$, como se muestra en la figura 3.

La ausencia de práctica deportiva se encontró igualmente asociada al consumo de tabaco con una diferencia estadísticamente significativa $(p<0,001)$, como muestra la figura 4 , en la que se observa una mayor proporción de chicos que no practican ningún deporte en el grupo de fumadores.

El análisis del rendimiento escolar, utilizando el número de asignaturas aprobadas en la última evaluación, mostró que el $81,8 \%$ de los fumadores había aprobado 9 asignaturas o menos de las 11 que componían su currículo. En el grupo de no fumadores fue mayor la proporción de chicos que habían aprobado $10 \mathrm{u}$ 11 asignaturas, descendiendo la fracción de aquéllos que habían aprobado 9 o menos al 47,3\% ( $<<0,001)$.

El consumo de alcohol se mostró igualmente relacionado con el hábito de fumar como se muestra en la figura 5, donde se puede apreciar la mayor proporción de consumidores de alcohol en el grupo de fumadores $(p<0,001)$.
Finalmente, mediante regresión logística, se comprobó que las variables asociadas de manera independiente con el consumo de tabaco en los escolares de $1^{\circ}$ de E.S.O. son: tener algún amigo fumador, el consumo de alcohol, la ausencia de práctica deportiva, la mayor edad y el menor nivel de estudios del padre. En la tabla 1 se observan estas variables con sus odds ratio e intervalos de confianza correspondientes y en la figura 6 se muestra una representación gráfica comparativa de dichas variables.

\section{DISCUSIÓN}

En nuestro estudio identificamos diversos factores de exposición relacionados con el estilo de vida y el entorno sociofamiliar que se muestran asociados al hábito de fumar en la población escolar del primer curso de la enseñanza secundaria, la cual comprende habitualmente la adolescencia temprana. El hábito de fumar en algún amigo, el consumo de alcohol, una menor dedicación a la práctica deportiva de manera habitual, la mayor edad (14-15 años) y el menor nivel de estudios alcanzado por el padre fueron los factores que se asociaron de forma independiente al consumo de tabaco en esta población.

La encuesta se realizó en el marco de una actividad de prevención del tabaquismo en escolares ofrecida a numerosos colegios e institutos que imparten enseñanza secundaria en las provincias de Albacete y 
Ciudad Real y fue cumplimentada por todos los alumnos de aquellas clases que habían dado su consentimiento para participar. Dado que no se realizó una selección aleatoria de los participantes, la validez externa del estudio podría verse comprometida. Sin embargo, consideramos que la muestra puede ser representativa de la población de su misma edad dada la amplia distribución de los centros escolares participantes tanto en medio rural como urbano. Puesto que en nuestro país la escolarización es obligatoria hasta los 16 años, no es posible encontrar otro tipo de población de esa edad fuera de las aulas, aunque pudo existir una menor representación de los niveles socioculturales más elevados que hubieran escogido los centros privados para la escolarización. En nuestro estudio participó un único centro privado, siendo públicos los 20 restantes.

Igualmente, debemos tener en cuenta que todos los resultados proceden de un cuestionario autocumplimentado y por tanto su validez depende de la veracidad de las respuestas, aunque se intentó facilitar ésta asegurando el anonimato en el cuestionario.

Al tratarse de un estudio transversal no podemos inferir causalidad entre el consumo de tabaco y los factores que aparecen asociados al mismo en nuestro estudio, sin embargo los resultados obtenidos coinciden ampliamente con los de otras investigaciones de diseño longitudinal en el mismo grupo de edad, tanto en nuestro medio como en otros países.

La influencia de los amigos está ampliamente documentada en la literatura como un factor de elevada importancia en el inicio de los adolescentes a determinadas actividades. En el caso de la experimentación con el tabaco y su paso posterior a consumo de forma regular, se ha documentado que el $80-85 \%$ de los adolescentes se inicia con los amigos. Aunque se podría argumentar que esta asociación puede deberse, al menos en parte, a un proceso de selección de los amigos en función de su conducta fumadora, lo cierto es que numerosos estudios encuentran que la conducta percibida entre iguales a favor del tabaquismo es un factor predictivo del inicio en este hábito en los adolescentes ${ }^{4-11}$.

Otras variables relacionadas con el consumo de tabaco en un primer análisis bivariante, como la conducta de fumar en padres y hermanos, no se mantienen asociadas en el modelo de regresión logística en nuestro estudio, aunque sí lo están en otros trabajos tanto de diseño transversal ${ }^{8-10}$ como longitudinal ${ }^{12}$.

Al igual que en nuestro estudio, otros autores han encontrado asociación entre el consumo de alcohol y el hábito de fumar en adolescentes, llegando a incluir esta variable entre los factores predictores del inicio en el tabaquismo $0^{5,7,13,14}$.
La ausencia de práctica deportiva se asocia en nuestro estudio de forma independiente al consumo de tabaco. En este sentido Kristjansson et al. observaron una asociación inversa entre la actividad deportiva y el consumo de tabaco en un estudio tranversal realizado en adolescentes islandeses, coincidiendo con Holmen et al. en Noruega, por lo que practicar algún deporte de manera habitual podría constituir un factor protector para el inicio en el tabaquismo ${ }^{8,15,16}$.

En nuestros resultados observamos que la conducta fumadora era más frecuente en el grupo de mayor edad (14-15 años frente a 12-13), asociación que se mantuvo en el análisis multivariante y que ha sido igualmente descrita por otros autores. Gómez Cruz et al. comprobaron, en un estudio longitudinal realizado en población escolar entre 14 y 20 años, que el riesgo de fumar aumenta conforme aumenta la edad en este grupo poblacional ${ }^{4}$. Estos datos contradicen, sin embargo, los obtenidos por Ariza et al. en un estudio prospectivo anterior donde se identifica a la edad menor de 15 años como factor predictivo del inicio en el consumo de tabaco ${ }^{7}$.

En cuanto al menor nivel de estudios del padre como factor asociado al consumo de tabaco en adolescentes, no encontramos investigaciones similares que sugieran esta asociación. Por el contrario, Rachiotis et al., en un estudio tranversal realizado en escolares griegos, encuentran que es el nivel de estudios medio o superior de los padres el que se asocia con el consumo de tabaco en el análisis bivariante, si bien esta asociación no se mantiene en el análisis multivariante y se trata de población escolar de un medio diferente ${ }^{17}$.

Llama la atención en nuestro estudio, por último, que el $64,4 \%$ de los estudiantes afirmara haber visto fumar a algún profesor a pesar de la prohibición de fumar en los centros escolares vigente en el momento de la encuesta. Este hecho podría deberse a que los alumnos recordaran la conducta fumadora del profesor en los meses previos a la entrada en vigor de las medidas legales restrictivas o bien al hecho de que los alumnos siguieran observando la conducta si el profesor acostumbra a fumar en la puerta del centro escolar, fuera del recinto.

\section{BIBLIOGRAFÍA}

1. Córdoba García R, Clemente Jiménez ML, Bartolomé Moreno C, García Suárez A. Importancia de que no se fume. En: Grupo de Abordaje del Tabaquismo de la semFYC. Manual de Tabaquismo. Barcelona: semFYC ediciones; 2008. p. 17-28.

2. Bandrés Gimeno R, Bello Rodríguez H, Castañal Canto X, Domínguez Grandal F. Tabaquismo pasivo. En: Grupo de Abordaje del Tabaquismo de la semFYC. Manual de Tabaquismo. Barcelona: semFYC ediciones; 2008. p. 63-82.

3. Márquez Pérez FL, Garrido Romero JJ, Domínguez Moreno JL. El consumo de tabaco como un proceso. En: Jimenez-Ruiz 
CA y Fagerström KO editores. Tratado de Tabaquismo. $2^{\mathrm{a}} \mathrm{ed}$ Majadahonda (Madrid): Ergon; 2007. p. 31-43.

4. Gómez Cruz G, Barrueco Ferrero M, Maderuelo Fernández A, Aparicio Coca I, Torrecilla García M. Factores predoctores de la conducta fumadora en alumnos de enseñanza secundaria. An Pedriatr (Barc) 2008; 68(5):454-61.

5. Caballero-Hidalgo A, González B, Pinilla J, Barber P. Factores predictores del inicio y consolidación del consumo de tabaco en adolescentes. Gac Sanit 2005; 19(6):440-7

6. Díaz E, Villalbí JR, Nebot M, Aubà J, Sanz F. El inicio de consumo de tabaco en escolares: estudio transversal y longitudinal de los factores predictivos. Med Clin (Barc) 1998; 110:334-9.

7. Ariza i Cardenal C, Nebot i Adell M. Predictores de la iniciación al consumo de tabaco en escolares de enseñanza secundaria de Barcelona y Lleida. Rev Esp Salud Pública 2002; 76:22738.

8. Kristjansson A, Sigfusdottir ID, Allegrante JP, Helgason AR. Social correlates of cigarette smoking among Icelandic adolescents: A population-based cross-sectional study. BMC Public Health 2008; 8:86.

9. Aubà J, Villalbí JR. Tabaco y adolescentes: influencia del entorno personal. Med Clin (Barc) 1993; 100:506-9.

10. Bolzán A, Peleteiro R. Tabaquismo durante la adolescencia temprana. Estudio en escolares argentinos. J Pediatr (Rio J) 2003; 79(5):461-6
11. Da Silva Pinto D, Aparecida Ribeiro S. Variables related to smoking initiation among students in public and private high schools in the city of Belém, Brazil. J Bras Pneumol 2007; 33(5):558-64

12. Nebot M, Tomás Z, Ariza C, Valmayor S, López MJ, Juárez $\mathrm{O}$. Factores asociados con el inicio del tabaquismo: seguimiento a los 3 años de una cohorte de escolares. Arch Bronconeumo 2004; 40(11):495-501.

13. Alcalá Cornide M, Azañas Ruiz S, Moreno Torres C, Gálvez Alcaraz L. Consumo de alcohol, tabaco y otras drogas en adolescentes, estudio de dos cohortes. Medicina de Familia (And) 2002; $2: 81-7$

14. Dee TS. The complementary of teen smoking and drinking. $J$ Health Econ 1999; 18(6):769:93.

15. Marrón Tundidor R, Pérez Trullén A, Clemente Jiménez ML, Herrero Labarga I. Factores de inicio del consumo de tabaco en adolescentes. Prevención del tabaquismo 2003; 5(4):219-34

16. Holmen TL, Barret-Connor E, Clausen J, Holmen J, Bjermer L. Physical exercice, sports and lung function in smoking versus nonsmoking adolescents. The Nord -Trondelag Study, Norway 1995-1997. Eur Respir J 2002; 19:8-15.

17. Rachiotis G, Muula AS, Rudatsikira E, Siziya S, Kyrlesi A, Gourgoulianis $\mathrm{K}$ et al. Factors associated with adolescent cigarette smoking in Greece: Results from a cross sectional study (GYTS Study). BMC Public Health 2008; 8:313. 